\title{
Corrections
}

\section{Correction: Goda et al., “Drosophila DH31 Neuropeptide and PDF Receptor Regulate Night-Onset Temperature Preference"}

In the article "Drosophila DH31 Neuropeptide and PDF Receptor Regulate Night-Onset Temperature Preference" by Tadahiro Goda, Xin Tang, Yujiro Umezaki, Michelle L. Chu, and Fumika Hamada, which appeared on pages 11739-11754 of the November 16, 2016 issue, authors Michael Kunst and Michael N. Nitabach were inadvertently omitted from the author list. The authors apologize for the oversight. The corrected author list is as follows: Tadahiro Goda, ${ }^{1}$ Xin Tang, ${ }^{1}$ Yujiro Umezaki, ${ }^{1}$ Michelle L. Chu, ${ }^{1}$ Michael Kunst, ${ }^{2}$ Michael N. Nitabach, ${ }^{2}$ and Fumika N. Hamada. ${ }^{1,3,4,5}$ Their affiliations are as follows: ${ }^{1}$ Visual Systems Group, Abrahamson Pediatric Eye Institute, Division of Pediatric Ophthalmology, Cincinnati Children's Hospital Medical Center, Cincinnati, Ohio 45229, ${ }^{2}$ Department of Cellular and Molecular Physiology, Department of Genetics, Kavli Institute for Neuroscience, Yale University School of Medicine, New Haven, Connecticut 06520, ${ }^{3}$ Division of Developmental Biology, Cincinnati Children's Hospital Medical Center, Cincinnati, Ohio 45229, ${ }^{4}$ Japan Science and Technology Agency, Precursory Research for Embryonic Science and Technology (PRESTO), Saitama 332-0012, Japan, and ${ }^{5}$ Department of Ophthalmology, College of Medicine, University of Cincinnati, Cincinnati, Ohio 45229. The author contributions footnote should have read "Author contributions: T.G. and F.N.H. designed research; T.G., X.T., Y.U., and M.L.C. performed research; M.K. and M.N.N. contributed unpublished reagents; T.G., X.T., Y.U., and F.N.H. analyzed data; and T.G. and J.C. wrote the paper." This correction does not affect the conclusions of the paper.

DOI: 10.1523/JNEUROSCI.0476-17.2017

\section{Correction: Wang et al., “T394A Mutation at the $\mu$ Opioid Receptor Blocks Opioid Tolerance and Increases Vulnerability to Heroin Self-Administration in Mice"}

In the article "T394A Mutation at the $\mu$ Opioid Receptor Blocks Opioid Tolerance and Increases Vulnerability to Heroin SelfAdministration in Mice” by Xiao-Fei Wang, Elisabeth Barbier, Yi-Ting Chiu, Yi He, Jia Zhan, Guo-Hua Bi, Hai-Ying Zhang, Bo Feng, Lee-Yuan Liu-Chen, Jia Bei Wang, and Zheng-Xiong Xi, which appeared on pages 10392-10403 of the October 5, 2016 issue, the authors regret that funding was omitted from the acknowledgments. The corrected acknowledgment is as follows: “This work was supported by the Intramural Research Program of the National Institute on Drug Abuse and National Institute on Drug Abuse Grants DA11925 and DA018722 to J.B.W. and P30 DA013429 to L.Y.L.-C.” This correction does not affect the conclusions of the paper.

DOI: $10.1523 / J N E U R O S C I .0482-17.2017$

\section{Correction: Walczak et al., "Novel Higher-Order Epigenetic Regulation of the Bdnf Gene upon Seizures"}

In the article "Novel Higher-Order Epigenetic Regulation of the Bdnf Gene upon Seizures" by Agnieszka Walczak, Andrzej A. Szczepankiewicz, Blazej Ruszczycki, Adriana Magalska, Katarzyna Zamlynska, Joanna Dzwonek, Ewa Wilsczek, Katarzyna ZyburaBroda, Marcin Rylski, Monika Malinowska, Michal Dabrowski, Teresa Szczepinska, Krzysztof Pawlowski, Marta Pyskata, Jakub Wlodarczyk, Izabela Szczerbal, Marek Switonski, Marion Cremer, and Grzegorz M. Wilczynski, which appeared on pages 2507-2511 of the February 6, 2013 issue, the authors regret a grant number was omitted from the acknowledgments. The corrected acknowledgment is as follows "This work was supported by Polish-Norwegian Grant PNRF-96-Al-1/07, and by the European Regional Development Fund POIG 01.01.02-00-008/08. The presented research was financed by “PRELUDIUM” grant number UMO-2011/03/N/NZ3/ 04516 financed by the National Center of Science." This correction does not affect the conclusions of the paper.

DOI: $10.1523 / J N E U R O S C I .0477-17.2017$ 